\title{
Clinical and Serologic Characteristics of Systemic Lupus Erythematosus in the Arab World: A Pooled Analysis of 3,273 Patients
}

\author{
Marwan ADWAN (D \\ Department of Medicine, The University of Jordan, Amman, Jordan
}

\begin{abstract}
Objectives: This study aims to describe the clinical and immunological characteristics of systemic lupus erythematosus (SLE) in the Arab world. Materials and methods: We searched PubMed and Google Scholar for observational studies describing the clinical and serologic features of SLE in adult patients in the Arab world. We used the search terms "lupus in Arabs" and the names of individual Arab countries. Twenty-two articles from 11 countries including 3,273 patients (349 males, 2,924 females; mean age 28.9 years) met the inclusion criteria and were analyzed. Studies that reported on either clinical or serologic data in adult patients were included.

Results: The mean age at disease onset was 28.9 years. The female to male ratio was 8.34:1. The most common clinical manifestations were arthralgia/arthritis (81.1\%), anemia (55.6\%), fatigue (53.4\%), malar rash (53.1\%) and renal manifestations (50.4\%). Antinuclear antibodies were present in $97.2 \%$, anti-double stranded deoxyribonucleic acid in $74.1 \%$, anti-Ro/Sjögren syndrome A in $50.5 \%$, anti-ribonucleoprotein in $43.5 \%$, anti-Smith in $40.7 \%$ and anti-La/Sjögren syndrome B in $29.2 \%$. The mortality rate was $7.6 \%$. The frequency of various clinical and immunological manifestations varied between different regions.

Conclusion: SLE displays several different clinical and serologic characteristics, both among different Arab populations and in comparison to other ethnic groups.

Keywords: Arab, ethnicity, lupus, Middle East, systemic lupus erythematosus.
\end{abstract}

Systemic lupus erythematosus (SLE) generally exhibits different phenotypic expressions and severities among different ethnic groups and populations. Data from observational studies support this variability. SLE in blacks, for instance, tends to be more common and more severe than in whites and is associated with an increased frequency of antibodies to Smith $(\mathrm{Sm})$ and ribonucleoprotein (RNP) antigens. ${ }^{1,2}$ Chinese, on the other hand, tend to have a high frequency of hemolytic anemia and anti-Ro/Sjögren syndrome A (SSA) antibodies. ${ }^{3,4}$ Latin Americans seem to have higher frequency of oral ulcers and myositis than white Europeans, but the reverse is true of vascular thrombosis. ${ }^{5,6}$

Lupus in the Arab world is quite common and may, in fact, be under-reported. Several studies describing the clinical and serologic features of SLE in individual Arab countries have been published, but there are no data on the clinical manifestations or immunologic characteristics of SLE from the Arab world as a whole. Furthermore, there is scarcity of prospective and multicenter studies that characterize the clinical features of lupus in Arabs. Therefore, in this study, we aimed to describe the clinical and

Received: January 11, 2018 Accepted: February 01, 2018 Published online: March 23, 2018

Correspondence: Marwan Adwan, MD. Department of Medicine, The University of Jordan, 11942 Amman, Jordan. Tel: +96265353666 e-mail: drm.adwan@yahoo.com 
immunological characteristics of SLE in the Arab world.

\section{MATERIALS AND METHODS}

We searched Pubmed and Google Scholar for observational studies describing the clinical and serologic features of SLE in adult patients in the Arab world. We used the search terms "lupus in Arabs" and the names of individual Arab countries. Data were collected at Jordan University Hospital, Department of Medicine between August 2016 and August 2017. Studies that reported on either clinical or serologic data in adult patients were included. Studies that included more than 20\% non-Arabs in their analysis were excluded, but studies that analyzed Arab subjects separately were included regardless of the proportion of non-Arabs they included. In such situations, only data on Arab patients were computed in the analysis. The study was conducted in accordance with the principles of the Declaration of Helsinki.

To minimize random error inherent in all small studies, we excluded studies involving less than 50 participants. We also excluded clinical trials, articles reporting on children and articles that dealt with only one aspect of lupus (renal, pulmonary or dermatologic etc.) and that did not include demographic or immunologic data. Data from all studies were pooled into a large sample.

We reviewed the full text articles of 39 studies. Seventeen studies were excluded (five sample sizes with lower than 50 participants, five involved one organ system, three genetic studies, one outcome study, one involved 50\% non-Arab ethnicities, one incidence and prevalence study, and one intensive care unit admission). Twenty-two articles ${ }^{7-28}$ from 11 countries including 3,273 patients (349 males, 2,924 females; mean age 28.9 years) met the inclusion criteria and were analyzed (Table 1). The study design was retrospective in 16 studies, cross-sectional in four studies and case-control in two studies.

\section{Statistical analysis}

Statistical analysis was performed with Statistical Package for the Social Sciences (version 24) and Microsoft Excel. Descriptive statistics were expressed as mean \pm standard deviation or percentages. For continuous variables (mean age at disease onset, mean age at diagnosis and mean disease duration), the means were merged and a weighted mean was computed taking into account the sample size of each study, using the following weighted mean equation:

$$
\mu(\text { weighted })=\frac{\left(\mu_{1} n_{1}+\mu_{2} n_{2}+\ldots+\mu_{i}(i) n_{i}\right) /}{\left(n_{1}+n_{2}+\ldots+n_{i}\right) .}
$$

(Where ' $\mu$ ' is the mean, ' $\mathrm{n}$ ' is the number of cases in each study and ' $i$ ' is the number of studies).

Percentages reported by individual studies for each manifestation were converted to absolute numbers. To compute a frequency for each manifestation, the numbers were merged and then reconverted to pooled percentages by dividing by the combined number of patients in all studies for each manifestation.

One study ${ }^{7}$ compared familial and sporadic SLE cases. In such case, we calculated the frequencies for both groups and combined them into one group.

After cross-tabulation and weighting by frequency, Fisher's exact test was used to compare the frequencies of various manifestations with those of two published cohorts. A $p$ value less than 0.05 was considered statistically significant.

\section{RESULTS}

The contributing countries were in descending order: Saudi Arabia with eight studies, Tunisia with three studies, Jordan with two studies, Kuwait with two studies, Egypt with one study, United Arab Emirates (UAE) with one study, Lebanon with one study, Oman with one study, Sudan with one study, Yemen with one study and Iraq with one study. The years of studies span the last three decades (from 1988 to 2016).

Of a total of 3,273 patients analyzed, females comprised $89.3 \%$ and males $10.7 \%$, giving a female to male ratio of $8.34: 1$. The number of patients in each study ranged from 50 to 749 (median 94) (Table 1). The mean disease duration 


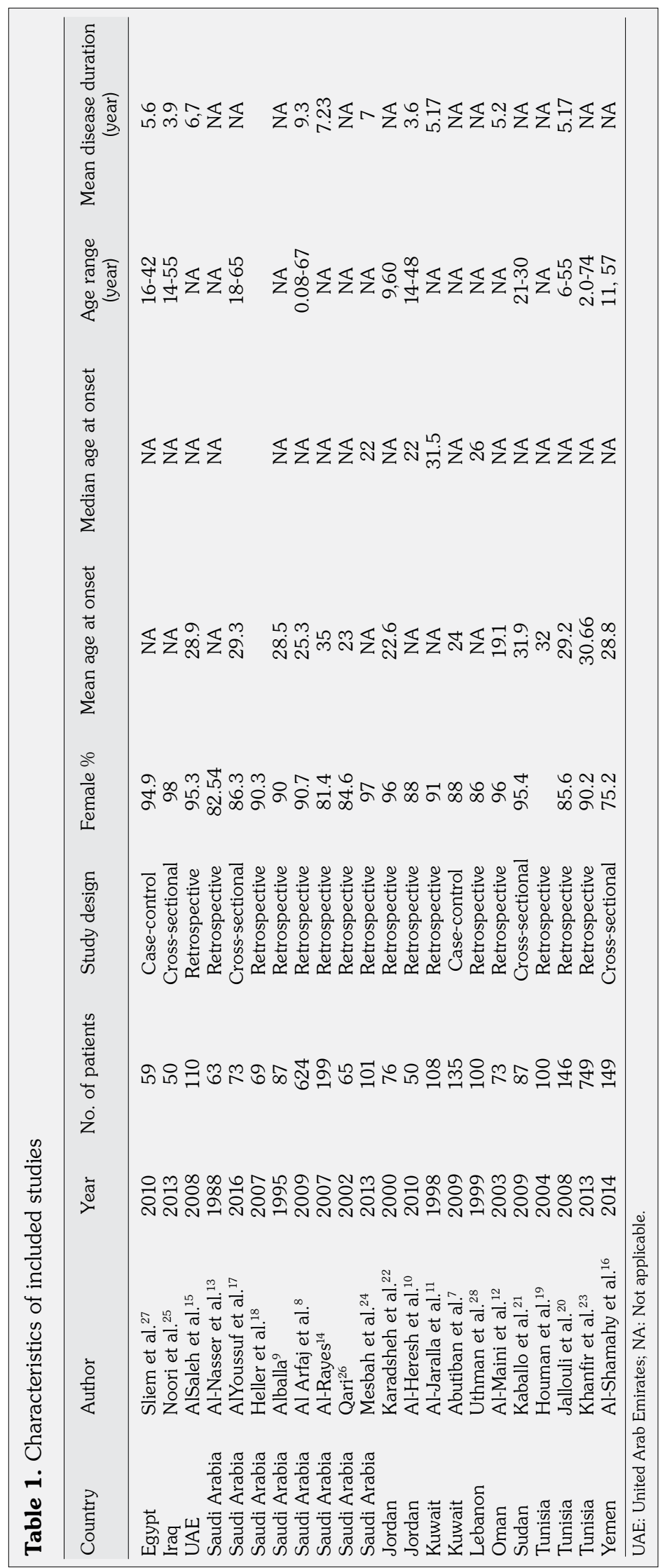




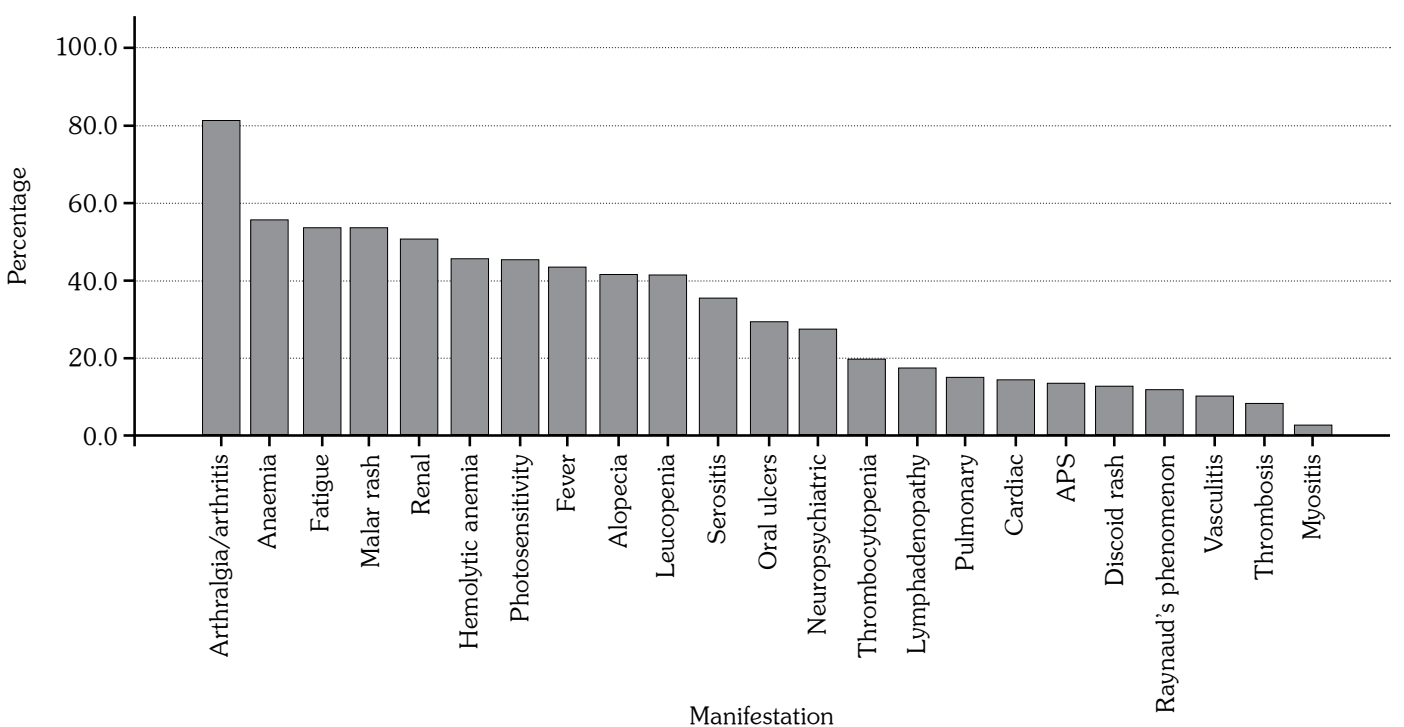

Figure 1. Frequency of systemic lupus erythematosus manifestations in Arab world. APS: Antiphospholipid syndrome.

was reported in eight studies and the average was $5.64 \pm 1.84$ years (range 4 to 9.3 years).

The pooled mean age at disease onset was 28.9 years. Only one study reported the mean age both at disease onset and at diagnosis. Nine studies reported the mean age at disease onset, but only five studies reported the mean age at diagnosis. Thus due to the small number, a weighted mean age at diagnosis was not

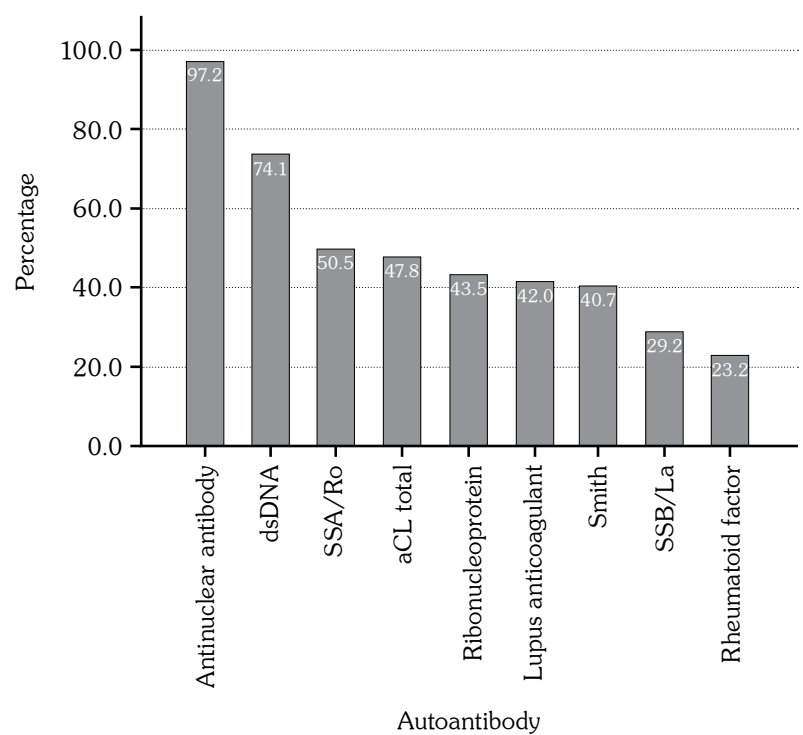

Figure 2. Frequency of autoantibodies in Arab patients with systemic lupus erythematosus. dsDNA: Anti-doublestranded deoxyribonucleic acid; SSA/Ro: Sjögren syndrome A/Ro; aCL: Anticardiolipin antibody; SSB/La: Sjögren syndrome B/La. calculated. Family history of SLE was reported in 116 of 1,399 patients (8.3\%).

The commonest clinical manifestation was arthralgia and/or arthritis, which occurred in $81.1 \%$. Other common manifestations included anemia (55.6\%), fatigue (53.4\%), malar rash (53.1\%) and renal manifestations (50.4\%). The least common manifestation was myositis, which was present in only $2.7 \%$. Figure 1 shows the combined frequencies of the various clinical manifestations.

Antinuclear antibodies (ANA) were present in the majority of cases (97.2\%). Anti-doublestranded deoxyribonucleic acid (anti-dsDNA) antibody was also common and was present in 74.1\%. Anti-Ro/SSA antibody was present in just over half of the patients. The frequency of other autoantibodies ranged from 23 to $40 \%$. Figure 2 shows the frequencies of the various immunological parameters.

Mortality was reported in nine studies and ranged from $3.1 \%$ to $15 \%$. Overall 117 deaths occurred among 1,676 patients, giving a mortality rate of $7.6 \%$. The causes of death were infection, active SLE, cardiac involvement, alveolar hemorrhage, pneumonitis, pulmonary embolism, pulmonary hypertension, renal failure, ruptured appendicitis, disseminated intravascular coagulation and leukemia. 
Table 2. Comparison of clinical and immunological features of systemic lupus erythematosus between different Arab countries

\begin{tabular}{|c|c|c|c|c|c|c|c|c|c|c|c|}
\hline & Egypt & Iraq & Jordan & Kuwait & Lebanon & Oman & Saudi & Sudan & Tunisia & UAE & Yemen \\
\hline Fatigue & & & & & & & 20.69 & 78.20 & & & 84.60 \\
\hline Fever & & & & 46.91 & & 31.30 & 33.80 & 66.70 & 2.11 & 45.90 & 81.90 \\
\hline Lymphadenopathy & & & & 5.35 & & & 11.24 & & & & \\
\hline Malar & 91.50 & & 49.21 & 34.98 & 52.00 & & 41.06 & 73.60 & 65.63 & 62.00 & 52.30 \\
\hline Discoid & 20.30 & & 10.32 & 7.00 & 19.00 & & 11.01 & 2.40 & 12.16 & 12.80 & \\
\hline Photosensitivity & 83.10 & & 25.40 & 39.92 & 16.00 & & 23.11 & 32.90 & 63.12 & 45.00 & 54.40 \\
\hline Alopecia & & & 30.16 & 48.15 & & 35.80 & 26.15 & & 3.12 & 55.00 & 49.00 \\
\hline Raynaud's phenomenon & & & & 19.34 & & & 4.22 & & 3.02 & & \\
\hline Oral ulcers & 84.70 & & 30.16 & 30.86 & 40.00 & 11.90 & 27.01 & 56.10 & 20.40 & 23.90 & \\
\hline Arthralgia arthritis & 52.54 & & 85.71 & 84.36 & 95.00 & 47.80 & 66.12 & 79.31 & 85.03 & 86.36 & 81.20 \\
\hline Serositis & 44.10 & & 10.32 & 25.93 & 40.00 & 23.90 & 20.14 & 34.10 & 47.04 & 16.50 & 9.40 \\
\hline Renal & 54.20 & 48.00 & 47.62 & 34.16 & 50.00 & 50.70 & 53.24 & 61.00 & 50.25 & 46.80 & 53.00 \\
\hline Neuropsychiatric & 30.50 & 20.00 & 24.60 & 20.99 & 19.00 & 33.80 & 25.84 & 13.40 & 34.57 & 15.60 & \\
\hline Cardiac & & 32.00 & & 4.53 & & 16.40 & 15.46 & & 30.15 & & \\
\hline Pulmonary & & & & 13.17 & & 7.50 & 18.42 & & 27.04 & & \\
\hline APS & & & 11.90 & 9.47 & & & 10.85 & & 9.35 & 15.60 & \\
\hline Thrombosis & & 20.00 & 8.73 & 7.41 & & & 4.14 & 4.60 & 7.24 & & \\
\hline Anaemia & 5.10 & & & & 10.00 & 64.10 & 39.89 & & 11.56 & 27.50 & 64.40 \\
\hline Thrombocytopenia & 33.90 & & 7.94 & 19.34 & 33.00 & 10.40 & 14.75 & 6.90 & 20.90 & 17.40 & 44.30 \\
\hline Leucopenia & & & & & & & & & & & \\
\hline lymphopenia & 55.90 & & 14.29 & 51.03 & 17.00 & 23.50 & 28.34 & 21.80 & 58.69 & 51.00 & 28.20 \\
\hline AIHA & & & 4.76 & 6.17 & 10.00 & & & 13.80 & 55.88 & 7.30 & \\
\hline ANA & 100.00 & 98.00 & 94.44 & 81.48 & 87.00 & 97.00 & 77.75 & 96.30 & 98.09 & 98.20 & 95.30 \\
\hline dsDNA & 72.88 & 56.00 & 81.75 & 46.50 & 50.00 & 91.78 & 66.82 & 51.72 & 74.17 & 85.45 & 59.73 \\
\hline $\mathrm{RF}$ & & & 19.05 & 3.29 & & 21.92 & 8.35 & & 3.02 & & \\
\hline RNP & & & & 6.58 & & 23.29 & 7.81 & & 44.32 & 39.09 & \\
\hline SSA_Ro & & & & 7.82 & & 43.84 & 9.13 & & 50.45 & 55.45 & \\
\hline SSB_La & & & & 4.94 & & 41.10 & 4.29 & & 27.24 & 21.82 & \\
\hline $\mathrm{Sm}$ & & 2.00 & 1.59 & 7.41 & & 50.68 & 14.75 & & 45.53 & & 27.52 \\
\hline LA & & 10.00 & 6.35 & & & & 6.01 & & 34.37 & 16.36 & \\
\hline $\mathrm{aCL}$ & & 20.00 & 7.14 & 7.00 & & 46.58 & 12.65 & & 62.91 & 21.82 & \\
\hline
\end{tabular}

Table 2 compares the various clinical and serologic features between various Arab countries. Fever ranged from 2.1\% (Tunisia) to $81.9 \%$ (Yemen). Malar rash ranged from 35\% (Kuwait) to $91.5 \%$ (Egypt). Discoid rash ranged from 2.4\% (Sudan) to 20.3\% (Egypt). Photosensitivity ranged from $16 \%$ (Lebanon) to $83.1 \%$ (Egypt). Alopecia ranged from $3.1 \%$ (Tunisia) to $55 \%$ (UAE). Oral ulcers ranged from $11.9 \%$ (Oman) to $84.7 \%$ (Egypt). Arthritis or arthralgia ranged from $47.8 \%$ (Oman) to 95\% (Lebanon). Serositis ranged from $9.4 \%$ (Lebanon) to $47 \%$ (Tunisia). Renal manifestations ranged from 34\% (Kuwait) to $61 \%$ (Sudan) Neuropsychiatric manifestations ranged from 13.4\% (Sudan) to 34.6\% (Tunisia). Cardiac manifestations ranged from $4.5 \%$ (Kuwait) to $32 \%$ (Iraq). Pulmonary manifestations ranged from $7.5 \%$ (Oman) to 27\% (Tunisia). Anemia ranged from $5.1 \%$ (Egypt) to $64.4 \%$ (Yemen). Thrombocytopenia ranged from 6.9\% (Sudan) to $44.3 \%$ (Yemen). Leucopenia/lymphopenia ranged from $14.3 \%$ (Jordan) to $58.7 \%$ (Tunisia). Hemolytic anemia ranged from $4.8 \%$ (Jordan) to 55.9\% (Tunisia).

Antinuclear antibodies ranged from $77.8 \%$ (Saudi) to $100 \%$ (Egypt). Anti-dsDNA ranged from 46.5\% (Kuwait) to $91.8 \%$ (Oman). Anti-RNP ranged from $6.6 \%$ (Kuwait) to $44.3 \%$ (Tunisia). Anti-Ro/SSA ranged from 7.8\% (Kuwait) to 55.5\% (UAE). Anti-La/Sjögren syndrome B (SSB) ranged from 4.3\% (Saudi) to $41.1 \%$ (Oman). Anti-Sm ranged from $1.7 \%$ (Jordan) to $50.7 \%$ (Oman). Lupus anticoagulant ranged from 6\% (Saudi) to 34.4\% (Tunisia). Anticardiolipin antibody (aCL) ranged from $7 \%$ (Kuwait) to $62.9 \%$ (Tunisia).

Table 3 shows the frequencies of the various clinical manifestations in comparison to two published cohorts (Euro-lupus ${ }^{5}$ and Grupo Latino Americano De Estudio del Lupus [GLADEL] ${ }^{6}$ ). There was no significant difference in the frequency 
Table 3. Clinical features of systemic lupus erythematosus in Arabs. Comparison with Europeans and Latin Americans

\begin{tabular}{|c|c|c|c|c|c|c|c|}
\hline Manifestation & Arab total & Arab No. & Arab \% & Europe \% & Latin American \% & Vs Europe & Vs Latin American \\
\hline Fever & 1883 & 821 & 43.6 & 52 & 56.7 & $<0.0001$ & $<0.0001$ \\
\hline Lymphadenopathy & 923 & 157 & 17 & 12 & 14.7 & 0.001 & 0.078 \\
\hline Malar rash & 3081 & 1642 & 53.3 & 58 & 61.3 & 0.006 & $<0.0001$ \\
\hline Discoid rash & 2670 & 339 & 12.7 & 10 & 11.8 & 0.031 & 0.227 \\
\hline Photosensitivity & 2819 & 1277 & 45.3 & 45 & 56.1 & 0.514 & $<0.0001$ \\
\hline Oral ulcers & 2842 & 834 & 29.3 & 24 & 41.7 & $<0.0001$ & $<0.0001$ \\
\hline Raynaud's phenomenon & 1113 & 132 & 11.9 & 34 & 28.2 & $<0.0001$ & $<0.0001$ \\
\hline Arthritis & 3024 & 2452 & 81.1 & 84 & 93.2 & 0.021 & $<0.0001$ \\
\hline Serositis & 2747 & 972 & 35.4 & 36 & 40.6 & 0.296 & 0.001 \\
\hline Renal & 3273 & 1651 & 50.4 & 39 & 51.7 & $<0.0001$ & 0.232 \\
\hline Neuropsychiatric & 3124 & 856 & 27.4 & 27 & 26.4 & 0.372 & 0.257 \\
\hline Cardiac & 2177 & 313 & 14.4 & - & 42.9 & - & $<0.0001$ \\
\hline Pulmonary & 2262 & 338 & 14.9 & 7 & 6.1 & $<0.0001$ & $<0.0001$ \\
\hline Myositis & 1165 & 31 & 2.7 & 9 & 17.5 & $<0.0001$ & $<0.0001$ \\
\hline Thrombosis & 2032 & 168 & 8.3 & 14 & 5.6 & $<0.0001$ & 0.003 \\
\hline Thrombocytopenia & 3078 & 607 & 19.7 & 22 & 19.2 & 0.066 & 0.364 \\
\hline Leucopenia & 3078 & 1273 & 41.4 & - & 42.3 & - & 0.290 \\
\hline Hemolytic anemia & 1331 & 607 & 45.6 & 8 & 11.8 & $<0.0001$ & $<0.0001$ \\
\hline Avascular necrosis & 897 & 25 & 2.8 & - & 1.1 & - & 0.003 \\
\hline
\end{tabular}

Table 4. Frequency of immunological manifestations among Arabs with lupus compared to Euro-lupus and Grupo Latino Americano De Estudio del Lupus cohorts

\begin{tabular}{lccccccc}
\hline & Number & Total & $\%$ & Euro-lupus \% & GLADEL \% & Vs Eurolupus & Vs GLADEL \\
\hline Antinuclear antibody & 2791 & 2870 & 97.2 & 96 & 97.9 & 0.082 & 0.126 \\
Anti-double stranded deoxyribonucleic acid & 2221 & 2996 & 74.1 & 78 & 70.5 & 0.009 & 0.016 \\
Rheumatoid factor & 185 & 796 & 23.2 & 18 & - & 0.004 & - \\
Low C3 & 683 & 1272 & 53.7 & - & 49.3 & - & 0.024 \\
Low C4 & 615 & 1174 & 52.4 & - & 53.8 & $<0.0001$ & 0.002 \\
Ribonucleoprotein & 595 & 1367 & 43.5 & 13 & 51.4 & $<0.0001$ \\
SSA/Ro & 713 & 1410 & 50.5 & 25 & 48.8 & $<0.0001$ & 0.521 \\
SSB/La & 392 & 1343 & 29.2 & 19 & 29.2 & $<0.0001$ \\
Smith & 720 & 1770 & 40.7 & 10 & 48.4 & -0.001 \\
Lupus anticoagulant & 450 & 1070 & 42.0 & 15 & 30.4 & - & 0.001 \\
Anti-cardiolipin antibody total & 882 & 1846 & 47.8 & - & - & - \\
Anti-cardiolipin antibody IgG & 202 & 517 & 39.1 & 24 & 50,6 & $<0.0001$ & $<0.0001$ \\
Anti-cardiolipin antibody IgM & 138 & 444 & 31.1 & 13 & 39.2 & $<0.0001$ & - \\
Anti-cardiolipin antibody unspecified & 632 & 1169 & 54.1 & - & - & - \\
Vs: Versus; C: Complement; SSA/Ro: Sjögren syndrome A/Ro; SSB/La: Sjögren syndrome B/La; Ig: Immunoglobulin; GLADEL: Grupo Latino Americano De \\
Estudio del Lupus; P<0.05 is considered statistically significant (Fisher's exact test).
\end{tabular}

of photosensitivity, serositis, neuropsychiatric manifestations or thrombocytopenia when compared to Euro-lupus cohort. Fever, malar rash, Raynaud's phenomenon, arthritis, thrombosis and myositis were less frequent than in the European cohort. Lymphadenopathy, discoid rash, oral ulcers, renal involvement, pulmonary and hemolytic anemia, on the other hand, were more common in Arabs than in Europeans. Hemolytic anemia particularly had a very high frequency $(45.6 \%$ vs. $8 \%$ Europeans vs. $11.8 \%$ Latin Americans).
Table 4 compares the frequencies of immunological parameters to Europeans and Latin Americans. There was no significant difference in the frequency of ANA between Arabs and Europeans or Latin Americans. With the exception of anti-dsDNA, all other autoantibodies were significantly more prevalent in Arabs than in Europeans. Anti-dsDNA was lower in Arabs than in Europeans but higher than in Latin Americans. When compared to Latin Americans, there was no difference in the frequency of anti-Ro/SSA or anti$\mathrm{La} / \mathrm{SSB}$. Lupus anticoagulant was higher in Arabs while anti-RNP, anti-Sm and aCL were lower. 


\section{DISCUSSION}

In this study, we collected data on the clinical and immunological features of SLE reported from eleven different Arab countries. We then synthesized the data and computed the frequencies of various clinical manifestations. In doing so, we took into account the sample size of each study and weighted the manifestations accordingly.

We analyzed data on 3,273 patients. The majority of patients were from Saudi Arabia and Tunisia (39\% and 30\%, respectively). The remaining $29 \%$ were from nine other countries. The female to male ratio was 8.34:1. This is slightly lower than in Europeans and Latin Americans (10.1:1 and 8.9:1, respectively). The pooled mean age at disease onset (28.9 years) was not different from Europeans or Latin Americans (29 and 28 years, respectively). Family history of SLE was present in $8.3 \%$.

The commonest clinical manifestation was arthritis or arthralgia, followed by anemia, fatigue, malar rash and renal manifestations. This pattern is similar to both Euro-lupus and GLADEL cohorts.

Most patients were positive for ANA, while anti-dsDNA was present in three quarters. Antibodies to extranuclear antigen, aCL and lupus anticoagulant (LA) were common (occurring in 40-50\%), although anti-La/SSB was present in only 29\%. Anti-Sm antibodies were extremely rare in Iraq and Jordan.

The mortality rate was $7.6 \%$ (3.1-15\%). However, there was no temporal pattern to the mortality or the five-year survival reported in the included studies to reflect the improved survival that took place over the past two decades. Therefore, the figures may not necessarily reflect the actual mortality in those countries and may simply represent a snapshot of the mortality rate in the institution from which they were reported. Furthermore, the reported mortality rates may not reflect the true overall mortality, as most studies reported hospital figures and did not take into account deaths in the community.

When we stratified by country, there were obvious differences between countries in relation to the different manifestations. Thus, fever was very rare in Tunisia (2.1\%) and very common in Yemen (81.9\%). Apart from these two extremes, the frequency of fever ranged from 33 to $67 \%$. Malar rash was particularly common in Egyptians, affecting 91.5\%. Discoid rash was surprisingly very uncommon in Sudan. Photosensitivity was least common in Lebanon and most common in Egypt. Alopecia was very rare in Tunisia. Oral ulcers were most common in Egypt. Serositis was rare in Yemen and Jordan and very common in Tunisia and Egypt. With the exception of Kuwait, renal manifestations were common in all countries (54.4\%) and significantly more frequent than in Europe. Neuropsychiatric manifestations affected a third of patients from Oman, Egypt and Tunisia and were least common in Sudan and UAE. Cardiac manifestations were particularly common in Iraq and Tunisia and rather uncommon in Kuwait. Hemolytic anemia was uncommon in the Arab world ranging from 4.8 to $13.8 \%$ and was comparable to Euro-lupus and GLADEL cohorts. The exception was Tunisia, where hemolytic anemia was reported in over half of the patients. This has resulted in a statistically significant difference in the frequency of hemolytic anemia compared to Europeans and Latin Americans. These inter-state differences may be due to the heterogeneity of the Arab peoples. The Tunisian study did not in fact state clearly the proportion of Arabic subjects. The ethnic composition of Tunisia which has more Berbers than Arabs may in part account for these significant differences. ${ }^{29}$ Therefore, it should be assumed that hemolytic anemia is rare in Arabs generally but very common in Tunisians.

When compared to Europeans, Arabs have a significantly less frequency of thrombosis. This is not explained by the presence of antiphospholipid antibodies (aPL), as these are more common in Arabs. This lower risk of thrombosis despite a higher prevalence of aPL needs further evaluation in larger studies.

Myositis also appears rare in Arabs compared to other ethnicities. Again, this is not in keeping with the high prevalence of anti-RNP and antiRo/SSA in Arabs.

This study also revealed a significant difference in the immunological manifestations of SLE compared to those in European subjects. With the exception of ANA, all other autoantibodies 
(rheumatoid factor, RNP, Ro, La, Sm, aCL \& LA) were significantly more frequent in Arabs than in Europeans. Anti-dsDNA, on the other hand, was significantly lower in Arabs than in Europeans. When compared with Latin Americans, on the other hand, there was no significant difference with regards to ANA, anti-Ro/SSA, anti-La/SSB or complement 4 consumptions. There was a significant difference, however, with regards to other autoantibodies. Thus anti-dsDNA and low complement 3 were more frequent than in Latin Americans but anti-RNP, anti-Sm and aCL were less frequent. LA was significantly higher in Arabs than in Europeans and Latin Americans as 42\% of the patients in our study were positive for LA compared to $15 \%$ Europeans and 30.4\% Latin Americans.

This study has some limitations. Firstly, the retrospective nature of the original studies and the short period of analysis pose some limitations. Secondly, while combining data from several studies increased the power of our study, there is some heterogeneity among Arabs from different regions and even within the same country, and this needs to be taken into account. ${ }^{30,31}$ The lack of availability of data from several countries (Libya, Syria, Bahrain, Algeria, Morocco and Qatar) may also have affected our findings.

In conclusion, lupus in Arabs shares many similarities with that in other ethnic groups. At the same time, it displays several different clinical and immunologic characteristics, both among different Arab populations and in comparison to other ethnic groups. This study revealed a very high frequency of hemolytic anemia among Tunisian patients compared to other Arab and non-Arab populations. Anti-extractable nuclear antigen antibodies (Ro, La, RNP, Sm) are present in a larger number of patients than in Europe and show a similar distribution to that seen in South Americans. Mucocutaneous features seem to be particularly common in Egyptian patients, although this should be taken with caution as the Egyptian data are based on one small study of 59 patients. Larger studies are needed to clarify this point. Finally, our study revealed that myositis is uncommon among Arabs with lupus.

\section{Declaration of conflicting interests}

The author declared no conflicts of interest with respect to the authorship and/or publication of this article.

\section{Funding}

The author received no financial support for the research and/or authorship of this article.

\section{REFERENCES}

1. Mody GM, Parag KB, Nathoo BC, Pudifin DJ, Duursma J, Seedat YK. High mortality with systemic lupus erythematosus in hospitalized African blacks. $\mathrm{Br}$ J Rheumatol 1994;33:1151-3.

2. Arnett FC, Hamilton RG, Roebber MG, Harley JB, Reichlin M. Increased frequencies of Sm and nRNP autoantibodies in American blacks compared to whites with systemic lupus erythematosus. J Rheumatol 1988;15:1773-6.

3. Mok CC, Lau CS. Lupus in Hong Kong Chinese. Lupus 2003;12:717-22.

4. Boey ML, Peebles CL, Tsay G, Feng PH, Tan EM. Clinical and autoantibody correlations in Orientals with systemic lupus erythematosus. Ann Rheum Dis 1988;47:918-23.

5. Cervera R, Khamashta MA, Font J, Sebastiani GD, Gil A, Lavilla P, et al. Systemic lupus erythematosus: clinical and immunologic patterns of disease expression in a cohort of 1,000 patients. The European Working Party on Systemic Lupus Erythematosus. Medicine (Baltimore) 1993;72:113-24.

6. Pons-Estel BA, Catoggio LJ, Cardiel MH, Soriano ER, Gentiletti S, Villa AR, et al. The GLADEL multinational Latin American prospective inception cohort of 1,214 patients with systemic lupus erythematosus: ethnic and disease heterogeneity among "Hispanics". Medicine (Baltimore) 2004;83:1-17.

7. Abutiban F, Mokkaddam K, Ameen E, Moussa M, Alsaeid K. Comparison between familial and sporadic systemic lupus erythematosus in Kuwaiti patients. Lupus 2009;18:86-91.

8. Al Arfaj AS, Khalil N. Clinical and immunological manifestations in 624 SLE patients in Saudi Arabia. Lupus 2009;18:465-73.

9. Alballa SR. Systemic lupus erythematosus in Saudi patients. Clin Rheumatol 1995;14:342-6.

10. Al-Heresh AM. Systemic Lupus Erythematosus among Jordanians: A Single Rheumatology Unit Experience. JRMS 2010;17:20-4.

11. Al-Jarallah K, Al-Awadi A, Siddiqui H, Al-Salim I, Shehab D, Umamaheswaran I, et al. Systemic lupus erythematosus in Kuwait--hospital based study. Lupus 1998;7:434-8.

12. Al-Maini MH, El-Ageb EM, Al-Wahaibi SS, Al-Farsi Y, Richens ER. Demographic, autoimmune, and clinical 
profiles of patients with systemic lupus erythematosus in Oman. Rheumatol Int 2003;23:186-91.

13. Al-Nasser A-AN, El-Shabrawy Aboul-Enein M, Al-Aska A-K. Systemic Lupus Erythematosus in Riyadh, Saudi Arabia. JRMS1988;108:90-3.

14. Al-Rayes H, Al-Swailem R, Arfin M, Sobki S, Rizvi S, Tariq M. Systemic lupus erythematosus and infections: a retrospective study in Saudis. Lupus 2007;16:755-63.

15. AlSaleh J, Jassim V, ElSayed M, Saleh N, Harb D. Clinical and immunological manifestations in 151 SLE patients living in Dubai. Lupus 2008;17:62-6.

16. Al-Shamahy HA, Dhaifallah NH, Al-Ezzy YM. Clinical and laboratory manifestations of yemeni patients with systemic lupus erythematosus. Sultan Qaboos Univ Med J 2014;14:80-7.

17. Alyoussuf A, Alassar B, Mohammed O, Mirghani $\mathrm{H}$, Amirthalingam P. Pattern of Systemic Lupus Erythematosus in Tabuk, Saudi Arabia. IJBAMR 2016;5:23-8.

18. Heller T, Ahmed M, Siddiqqi A, Wallrauch C, Bahlas S. Systemic lupus erythematosus in Saudi Arabia: morbidity and mortality in a multiethnic population. Lupus 2007;16:908-14.

19. Houman MH, Smiti-Khanfir M, Ben Ghorbell I, Miled M. Systemic lupus erythematosus in Tunisia: demographic and clinical analysis of 100 patients. Lupus 2004;13:204-11.

20. Jallouli M, Frigui M, Hmida MB, Marzouk S, Kaddour N, Bahloul Z. Clinical and immunological manifestations of systemic lupus erythematosus: study on 146 south Tunisian patients. Saudi J Kidney Dis Transpl 2008;19:1001-8.

21. Kaballo BG, Wahab AA, Nur MM, Modawi KA, Elnazir $\mathrm{E}$, Omer $\mathrm{H}$, et al. Ethnic distribution and clinical features of systemic lupus erythematosus in the Sudan. Sudan Med J 2009;45:49-57.

22. Karadsheh MF, Nimri FA, Ajlouni YM, Dneibat WA,
Karadsheh RF. The characteristics of systemic lupus erythematosus. A study in a general hospital. Saudi Med J 2000;21:282-6.

23. Khanfir MS, Houman $\mathrm{MH}$, Cherif E, Hamzaoui A, Souissi S, Ben Ghorbel I, et al. TULUP (TUnisian LUPus): a multicentric study of systemic lupus erythematosus in Tunisia. Int $\mathrm{J}$ Rheum Dis 2013;16:539-46.

24. Mesbah MR, Atwa E, Meshi M. A retrospective study of systemic lupus erythematosus (SLE) in Jazan: Clinical and immunological overview. Egyptian Journal of Medical Microbiology 2013;22:73-81.

25. Noori AS, Jawad AM, Jassim NA, Gorial FI. Prevalence of antiphospholipid antibodies in sample of Iraqi patients with systemic lupus erythematosus: A cross sectional study. American Journal of Clinical Medicine Research. 2013;1:61-4.

26. Qari FA. Clinical pattern of systemic lupus erythematosus in Western Saudi Arabia. Saudi Med J 2002;23:1247-50.

27. Sliem H, Tawfik G, Khalil KA, Ibrahim N. Pattern of systemic lupus erythematosus in Egyptian patients: the impact of disease activity on the quality of life. Pan Afr Med J 2010;6:14. Epub 2010 Aug 23

28. Uthman I, Nasr F, Kassak K, Masri AF. Systemic lupus erythematosus in Lebanon. Lupus 1999;8:713-5.

29. Hajjej A, Almawi WY, Hattab L, El-Gaaied A, Hmida S. HLA Class I and Class II Alleles and Haplotypes Confirm the Berber Origin of the Present Day Tunisian Population. PLoS One 2015;10:e0136909.

30. Almawi WY, Busson M, Tamim H, Al-Harbi EM, Finan RR, Wakim-Ghorayeb SF, et al. HLA class II profile and distribution of HLA-DRB1 and HLA-DQB1 alleles and haplotypes among Lebanese and Bahraini Arabs. Clin Diagn Lab Immunol 2004;11:770-4.

31. Hafez M, el-Shennawy FA. HLA-antigens in the Egyptian population. Forensic Sci Int 1986;31:241-6. 